\title{
Misdiagnosis of a chin abscess in a teenager with rhabdomyosarcoma - consequences for the patient's health and quality of life
}

\author{
Małgorzata Styczewska', Małgorzata A. Krawczyk², Ewa Bień2 \\ 'The English Division Pediatric Oncology Scientific Circle, Medical University of Gdansk, Gdansk, Poland \\ 2Department of Paediatrics, Haematology, and Oncology, Medical University of Gdansk, Gdansk, Poland
}

\section{ABSTRACT}

Childhood rhabdomyosarcoma (RMS) develops frequently within head and neck (H\&N) structures. Its clinical manifestation may mimic more common benign, inflammatory, and congenital lesions.

A 16-year-old boy with painless purple swelling of the chin soft tissues was treated with local and systemic antibiotics. Because no improvement was achieved, the growing "abscess" was incised and drained many times in the surgical department. After three months of ineffective therapies, the patient was transferred to the clinical hospital, where a tumour infiltrating the chin and oral cavity floor, impairing speech and swallowing, was detected. Alveolar RMS was diagnosed by histopathology of the tumour biopsy. Due to unfavourable prognostic factors, the patient was classified to a very high-risk group and received aggressive long-lasting oncological treatment. Although he achieved full remission, the sequelae of therapy affect his quality of life. Despite its rarity, RMS should be included in the differential diagnosis of H\&N lesions, especially painless and rapidly growing.

\section{KEY WORDS:}

abscess, RMS, rhabdomyosarcoma, misdiagnosis, head and neck.

\section{INTRODUCTION}

Rhabdomyosarcoma (RMS) is the most common subtype of soft tissue sarcoma (STS) in children, accounting for more than half of them. It is diagnosed in approximately 4.5/1,000,000 children per year, constituting around $4.5 \%$ of all paediatric malignancies $[1,2]$.

According to the current World Health Organization (WHO) classification of tumours of soft tissue and bone [3], four main histological subtypes of RMS are distinguished, including: embryonal, alveolar, pleomorphic, and spindle cell RMS. The first two are the most prevalent in children. Their biology, aggressiveness, response to chemotherapy (CHT), age predilection, and typical localisation differ significantly. Embryonal RMS (RME) is usually diagnosed in localised stage and develops in favourable anatomical sites; therefore, it is associated with a relatively good prognosis [4]. Conversely, alveolar RMS (RMA) is characterised by aggressive clinical behaviour, tendency to early dissemination via lymphatic and haematogenous routes, and, consequently, with unfavourable outcomes [5]. Such an aggressive biology of RMA may be attributed to the presence of gene fusions PAX3/FOXO1 and PAX7/ FOXO1. The prognosis in patients with tumours express-

\section{ADDRESS FOR CORRESPONDENCE:}

Małgorzata Styczewska, The English Division Paediatric Oncology Scientific Circle, Medical University

of Gdansk, 7 Dębinki St., 80-952 Gdansk, Poland, ORCID: 0000-0001-8280-1319,

e-mail: mstyczewska@gumed.edu.pl 
ing one of these translocations is significantly poorer than in patients with fusion-negative RMA $[6,7]$.

The head and neck $(\mathrm{H} \& \mathrm{~N})$ region is the most common anatomical location of paediatric RMS, being the primary tumour site in approximately $35 \%$ of cases [8]. RMS of the H\&N have been divided into three clinical subgroups: orbital, parameningeal, and non-parameningeal RMS [8]. Orbital RMS are usually of embryonal subtype, localised and associated with the most favourable prognosis [9]. Children with orbital RMS usually present with exophthalmos, often with concomitant eyeball immobilisation, an asymmetric eyelid infiltration, or strabismus [10]. Parameningeal RMS involves the nasopharynx, paranasal sinuses, parapharyngeal space, pterygopalatine and infratemporal fossae and middle ear. The symptomatology is unspecific with purulent or bloody discharges from the nose or ear and auricular and nasal duct obstruction predominating [10]. RMA histology and limited feasibility of complete resection result in poor prognosis of parameningeal RMS [11]. Non-parameningeal RMS comprise tumours developing within the oral cavity, nasal cavity, larynx, ear, cheeks, and salivary glands, as well as the skin, subcutaneous tissue and muscles of the H\&N. They typically present as a firm, non-painful, non-movable mass, disfiguring the area of H\&N. They are usually of embryonal histology and are more amenable to complete resection compared to parameningeal RMS, therefore carry more favourable prognosis [12].

Despite significant progress in the treatment of paediatric RMS over the last decades, early diagnosis in a low, resectable stage is still the most favourable prognostic factor. Therefore, all physicians need to include RMS in the differential diagnosis of more common diseases developing within the $\mathrm{H} \& \mathrm{~N}$.

\section{CASE REPORT}

A 16-year-old boy, a professional junior volleyball player, developed a $1.3 \mathrm{~cm} \times 1 \mathrm{~cm}$ purple painless swelling of the skin of the mental area. Initially he considered it as an acne eruption. Within a few days, the size of the lesion increased, so the patient went to a general practitioner, who ordered local therapy with antibiotic ointment (bacitracin and neomycin). The lesion continued to grow, so the patient was consulted by a dermatologist. At that time the purple lesion involved most of the chin, was elevated and painless. The regional lymph nodes were not enlarged and not painful. The dermatologist diagnosed superinfected acne and prescribed an oral antibiotic (clindamycin) to be administered for 14 days. After seven days of ineffective therapy the patient was referred to the surgical ward of the regional hospital with suspicion of an abscess of the chin. At that time the lesion involved the whole mental region and was extending towards the floor of the oral cavity, causing slight difficulty in speaking and swallowing. The lesion was not painful and neither fever nor enlarged cervical lymph nodes were present.

The surgeons introduced clindamycin intravenously. The "abscess" was incised; however, no pus was obtained but only blood. The discharge was sent for microbiological test and was found sterile. The results of laboratory tests were within normal ranges, except mildly elevated lactate dehydrogenase serum concentration.

During the next three weeks the lesion was drained several times without clinical improvement (Fig. 1). No ultrasound (USG) examination was performed. Eventually, the child was transferred to a clinical hospital for further imaging diagnostics and therapy. At the Department of Maxillo-Dental Surgery, magnetic resonance (MRI) of the head and neck region was performed. It revealed a pathological soft tissue mass measuring $46 \mathrm{~mm} \times$ $24 \mathrm{~mm} \times 42 \mathrm{~mm}$, involving soft tissues of the mental area adjacent to the mandible, extending to the oral cavity floor, with intensive contrast enhancement. The bone structures were not involved (Fig. 2 and 3). Apart from an unclear image of lymph node located inside the right

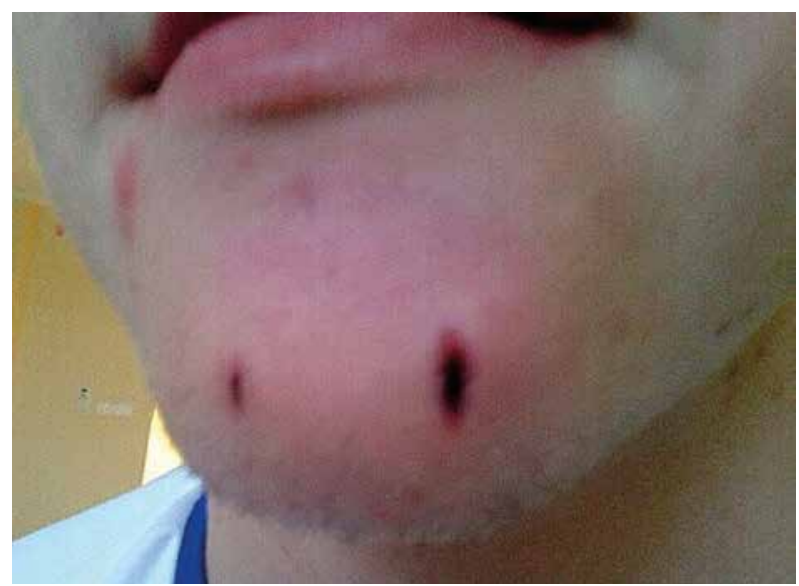

FIGURE 1. The lesion of the chin after incisions and drainage at the surgical department

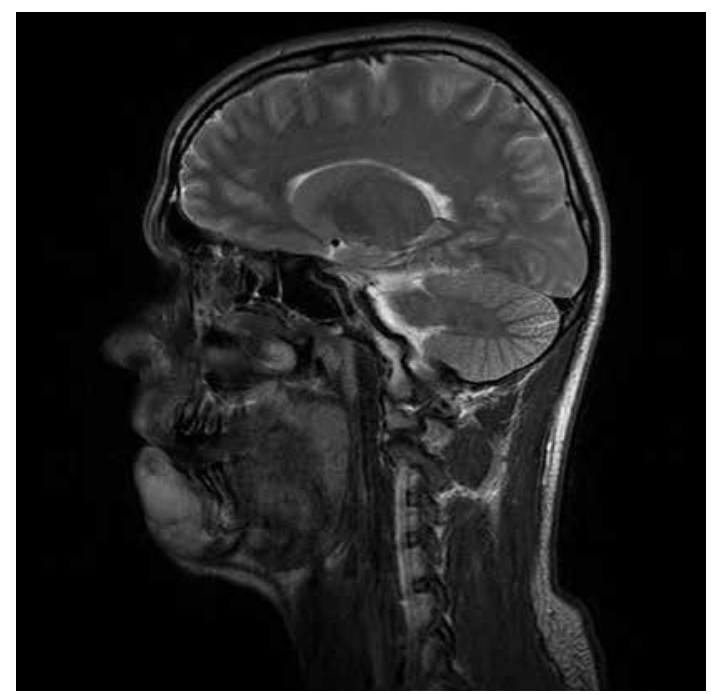

FIGURE 2. MRI of the head: hyperintensive solid mass on sagittal T2-weighted imaging located anterior to mandible 


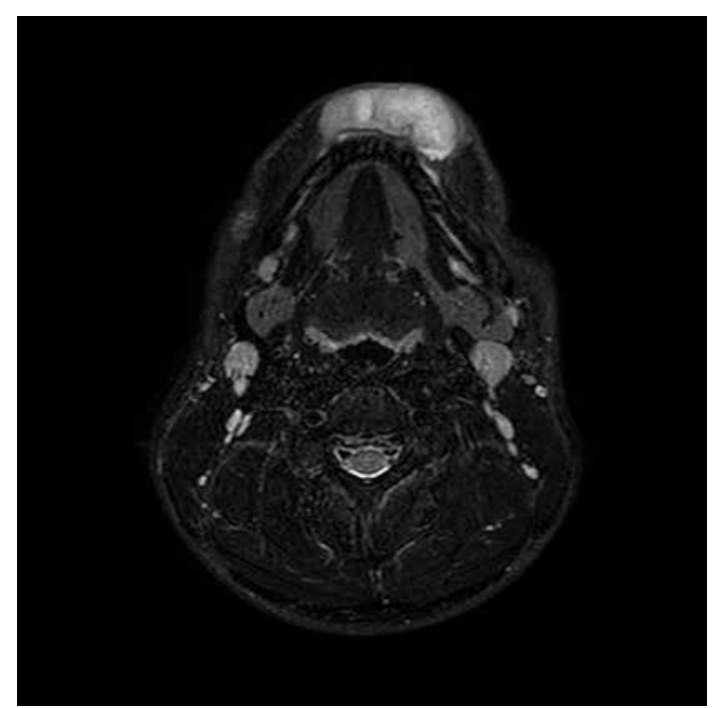

FIGURE 3. MRI of the head: axial T2-weighted image with fat saturation presenting focal, soft-tissue mass anterior to mandible

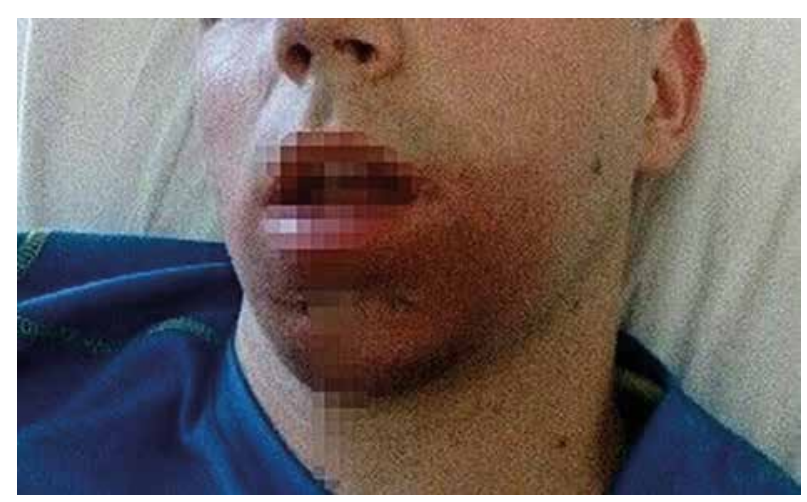

FIGURE 4. Large purple swelling of the soft tissues below the lower lip disfiguring the chin and submandibular area: the appearance of rhabdomyosarcoma at admission to the paediatric oncology department

parotid gland, no regional or distant metastases were detected in imaging studies. An open biopsy was taken, which revealed RMA histology. An examination towards the presence of gene fusions was not performed.

The boy was immediately referred to the Department of Paediatrics, Haematology, and Oncology, where further diagnostic procedures assessing the disease stage were performed. Finally, the patient was qualified to stage T2bNxM0 according to TNM classification and stage III according to IRS postsurgical system (Fig. 4).

Due to the uncertain image of enlarged lymph node within parotic gland $(\mathrm{Nx})$, it was difficult to decide whether to qualify the patient to the high-risk (HR) group (N0) or very high-risk (VHR) group (N1) of Cooperative Weichteilsarkom Study Group (CWS) protocol. Due to extremely high risk of local dissemination resulting from previous multiple surgical interventions within the tumour, the patient was individually qualified to the VHR group. Intense multidrug CHT was started immediately. The tumour responded well to $\mathrm{CHT}$, which allowed microscopically complete delayed excision of the remaining mass. Following tumour resection, radical radiotherapy (RTX) on the affected area with a total dose of 41.4 Gy was performed. Afterwards, the patient continued intense CHT (up to nine cycles) followed by eight cycles of oral maintenance CHT. He finished oncological therapy in full clinical remission of the disease. Currently, he remains disease-free, with a follow-up of 22 months since therapy discontinuation.

\section{DISCUSSION}

The symptoms of non-parameningeal RMS in children occur relatively early - most frequently in the form of a visible/palpable painless tumour of soft tissues or as cranial nerve palsies $[11,13]$. These symptoms are usually easier to detect early than the symptoms of parameningeal RMS, which are unspecific and misleading for a long time [14]. Accordingly, the prognosis in children with non-parameningeal RMS is better than for parameningeal RMS (10-year overall survival $74.2 \%$ vs. $66.1 \%)[15,16]$.

However, RMS developing in non-parameningeal $\mathrm{H} \& \mathrm{~N}$ region may also mimic other more common diseases of childhood, including post-traumatic haematoma, benign tumours, congenital malformations, and infectious processes. [12,17]. This was the case in our patient, in whom the suspicion of malignancy was raised in differential diagnosis during the three-month-long history of evolution of a non-painful purple swelling of soft tissues of the mental area.

Asymptomatic swelling is a typical presentation of RMS developing in non-parameningeal $\mathrm{H} \& \mathrm{~N}$ region. In a series of nine patients with RMS of the maxillofacial region, the first manifestation was painless or mildly symptomatic extraoral swelling in all but one child [11]. Likewise, the lesion of soft tissues of the mental area of our patient was never painful. The purple colour of the skin over the swelling was interpreted as symptom of the inflammatory process: at first as superinfected acne and then as an abscess. However, there were no symptoms typical for severe inflammation of soft tissues, including: pain, fever, enlarged regional lymph nodes and response to local and systemic antibiotics. Moreover, no pus was obtained after multiple surgical incisions and drainage of the suspected abscess. This information should immediately direct the path of differential diagnosis to causes other than inflammatory/purulent infiltration of soft tissues. Unfortunately, although during the three months preceding the proper oncological diagnosis the patient's lesion was consulted by a primary care physician, dermatologist, and surgeons, none of them suspected neoplasm. Also, none of these specialists ordered a USG examination of the lesion despite the fact that it was constantly growing. During the three-month-long interview, the size of the RMS of the chin increased from $1.2 \mathrm{~cm}$ to $4.6 \mathrm{~cm}$ in maximal diameter and gradually infiltrated not only the soft tissues of the mental area, but also the floor of 
the oral cavity, impairing speech and swallowing of saliva. This negatively impacted the disease stage and feasibility of performing a primary (upfront) surgical excision of the tumour.

The feasibility of complete tumour resection at the beginning of treatment is one of the key prognostic factors in childhood RMS, which implies the cancer stage. Non-parameningeal RMS of the H\&N, which are usually more amenable for radical resection, have better prognosis than parameningeal tumours [12]. However, in our patient, a non-mutilating radical excision of RMS was not feasible due to high local advancement of the disease found in clinical examination and in USG and MRI examinations performed at the paediatric oncology reference university centre. Therefore, only biopsy of the tumour was performed to obtain histopathological diagnosis.

Furthermore, numerous surgical incisions and drainages performed within the tumour substantially increased the risk of RMS dissemination via lymphatic and blood vessels. These unwanted surgical interventions put the patient in the VHR therapeutic group of the CWS protocol. To diminish the risk of metastases and treatment failure, very intense multidrug $\mathrm{CHT}$, including cardiotoxic anthracyclines (doxorubicin, idarubicin), was immediately started. It is important to note that the patient would be qualified to the lower risk group not treated with anthracyclines if the multiple tumour incisions had not been not made.

Orbach et al. reported the recurrence rate in non-parameningeal H\&N RMS to be as high as 50\% [18]. Because the vast majority of relapses are local/locoregional, the effective control of the primary tumour site is of utmost importance. In our patient the tumour size diminished significantly after neo-adjuvant CHT; however, to achieve R0 delayed tumour resection, the surgery had to be extensive and was associated with some aesthetic mutilation. The delayed resection involved not only the remaining tumour, but also all sites of previous surgical incisions and drainages (Fig. 5). Moreover, adjuvant RTX was administered, followed by eight courses of maintenance therapy to avoid local relapse and/or spread of RMA. The RTX field also had to cover all the sites of surgical interventions within the tumour; therefore, a much larger volume of radiation was necessary than if the diagnosis of RMA had been made earlier, without compromising the tumour tissue continuity. Long-lasting CHT and RTX helped to achieve complete remission of RMA; however, this can result in a high rate of late consequences, impairing the patient's quality of life, such as: second malignant neoplasms, hypothyroidism, cardiomyopathy, xerostomia, dental decay, and aesthetic discomfort [19].

Although eventually our patient survived the cancer, he is still at high risk of recurrence due to high probability of regional dissemination of RMA prior to diagnosis. Moreover, it should be emphasised that the oncological treatment was very long and exhausting. As a result, due

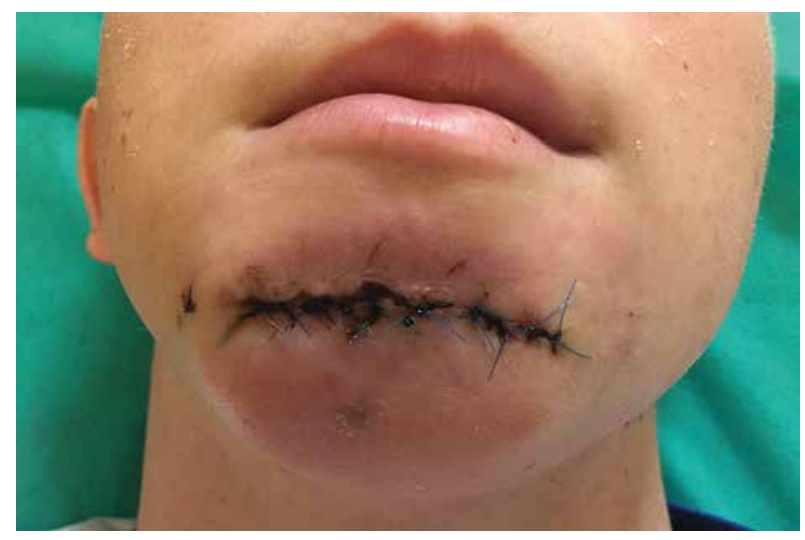

FIGURE 5. The scar after delayed complete resection of the tumour, covering all sites of previous surgical interventions

to reduced physical exercise tolerance, the patient lost the chance to join the prestigious professional volleyball team to which he had been accepted before his disease. The functional and aesthetic sequelae of surgery and RTX significantly impaired the patient's self-confidence.

Despite its rarity, RMS should be included in the differential diagnosis of masses in the $\mathrm{H} \& \mathrm{~N}$ area, especially if a non-painful, rapidly increasing lesion is observed. In doubtful cases, early imaging examination, including USG, and a consultation in a reference tertiary centre should be considered. Diagnostic biopsy of the tumour should be carefully planned by a paediatric oncologist and/or paediatric surgeon experienced in childhood cancers. To reduce delays in diagnosing malignancies (including RMS) of H\&N non-parameningeal area in children, constant education of paediatricians, family doctors, paediatric surgeons, maxillo-facial surgeons, laryngologists, and dermatologists is necessary.

\section{DISCLOSURE}

The authors declare no conflict of interest.

\section{REFERENCES}

1. Ray A, Huh WW. Current state-of-the-art systemic therapy for pediatric soft tissue sarcomas. Curr Oncol Rep 2012; 14: 311-319.

2. Radzikowska J, Kukwa W, Kukwa A, et al. Management of pediatric head and neck rhabdomyosarcoma: A case-series of 36 patients. Oncol Lett 2016; 12: 3555-3562.

3. Fletcher CDM, Bridge JA, Hogendoorn P, et al. WHO Classification of Tumours of Soft Tissue and Bone. 4th ed. 2013.

4. Perez EA, Kassira N, Cheung MC, et al. Rhabdomyosarcoma in children: A SEER population based study. J Surg Res 2011; 170: e243-251.

5. Scheer M, Dantonello T, Brossart P, et al. Importance of wholebody imaging with complete coverage of hands and feet in alveolar rhabdomyosarcoma staging. Pediatr Radiol 2018; 48: 648-657.

6. Williamson D, Missiaglia E, De Reyniès A, et al. Fusion gene-negative alveolar rhabdomyosarcoma is clinically and molecularly indistinguishable from embryonal rhabdomyosarcoma. J Clin Oncol 2010; 28: 2151-2158. 
7. Dasgupta R, Fuchs J, Rodeberg D. Rhabdomyosarcoma. Semin Pediatr Surg 2016; 25: 276-283.

8. Turner JH, Richmon JD. Head and neck rhabdomyosarcoma: A critical analysis of population-based incidence and survival data. Otolaryngol Head Neck Surg 2011; 145: 967-973.

9. Oberlin O, Rey A, Anderson J, et al. Treatment of orbital rhabdomyosarcoma: survival and late effects of treatment - results of an international workshop. J Clin Oncol 2001; 19: 197-204.

10. Radzikowska J, Kukwa W, Kukwa A, et al. Rhabdomyosarcoma of the head and neck in children. Contemp Oncol 2015; 19: 98-107.

11. Iatrou I, Theologie-Lygidakis N, Schoinohoriti O, et al. Rhabdomyosarcoma of the maxillofacial region in children and adolescents: Report of 9 cases and literature review. J Cranio-Maxillo-Facial Surg 2017; 45: 831-838.

12. Reilly BK, Kim A, Peña MT, et al.; Rhabdomyosarcoma of the head and neck in children: Review and update. Int J Pediatr Otorhinolaryngol 2015; 79: 1477-1483.

13. Pratt CB, Smith JW, Woerner S, et al. Factors leading to delay in the diagnosis and affecting survival of children with head and neck rhabdomyosarcoma. Pediatrics 1978; 61: 30-36.

14. Häußler SM, Stromberger C, Olze H, et al. Head and neck rhabdomyosarcoma in children: a 20-year retrospective study at a tertiary referral center. J Cancer Res Clin Oncol 2018; 144: 371-379.

15. Affinita MC, Ferrari A, Milano GM, et al. Long-term results in children with head and neck rhabdomyosarcoma: A report from the Italian Soft Tissue Sarcoma Committee. Pediatr Blood Cancer 2018; 65: e26876.

16. Merks JHM, de Salvo GL, Bergeron C, et al. Parameningeal rhabdomyosarcoma in pediatric age: Results of a pooled analysis from North American and European cooperative groups. Ann Oncol 2014; 25: 231-236.

17. Khanna G, Sato Y, Smith RJ, et al. Causes of facial swelling in pediatric patients: correlation of clinical and radiologic findings. Radiographics 2006; 26: 157-172.

18. Orbach D, Mosseri V, Gallego S, et al. Nonparameningeal head and neck rhabdomyosarcoma in children and adolescents: Lessons from the consecutive International Society of Pediatric Oncology Malignant Mesenchymal Tumor studies. Head Neck 2017; 39: 24-31.

19. Strojan P, Hutcheson KA, Eisbruch A, et al. Treatment of late sequelae after radiotherapy for head and neck cancer. Cancer Treat Rev 2017; 59: 79-92. 